\title{
Extrapolation to the Complete Basis Set Limit for Binding Energies of Noncovalent Interactions
}

\author{
Steve Scheiner* \\ Department of Chemistry and Biochemistry \\ Utah State University \\ Logan, UT 84322-0300
}

\begin{abstract}
A means of extrapolating from double and triple-valence basis sets to a complete basis set is examined in the context of the pnicogen bonds in the $\mathrm{BH}_{2} \mathrm{P} \cdots \mathrm{NH}_{3}$ complexes, with $\mathrm{B}=\mathrm{CH}_{3}, \mathrm{H}$, $\mathrm{NH}_{2}, \mathrm{CF}_{3}, \mathrm{OH}, \mathrm{Cl}, \mathrm{F}$, and $\mathrm{NO}_{2}$. Binding energies converge smoothly, and the trends for the various substituents B are unaffected by the basis set size, extrapolation, or level of inclusion of electron correlation, including MP2 and $\operatorname{CCSD}(\mathrm{T})$. The approach appears to be successful also for H-bonded systems, in particular the water dimer. In the event that full extrapolation within the context of $\operatorname{CCSD}(\mathrm{T})$ is not feasible, several more economical but attractive options are suggested, including a quantitative measure of the accuracy to be expected with each.
\end{abstract}

*email: steve.scheiner@usu.edu

keywords: pnicogen bond; MP2; CCSD(T); H-bond; counterpoise 


\section{INTRODUCTION}

Advances in computer technology and software development over the past decades continue to enhance the accuracy with which ab initio calculations can be applied to chemical problems. In contrast to the early years when most calculations were limited to the Hartree-Fock level, it has now become routine to add electron correlation to systems of even moderate size. Nonetheless, the ability to perform a full CI calculation, which incorporates all possible configurations, is not normally possible, and various methods have been developed to incorporate as much electron correlation as possible in an efficient manner. One strategy to deal with this problem has been the attempt to approach the full electron correlation energy in a controlled asymptotic manner [1-11].

Similarly, while many early computations were limited to small or even minimal basis sets, it is now common for researchers to employ multiple valence functions as well as large collections of polarization and very diffuse functions with small orbital exponents. Yet despite these advances, the goal of applying very large basis sets, approaching an infinite set, remains out of reach. This lingering shortcoming has motivated an array of efforts to approximate the results of a complete basis set via an asymptotic approach to infinity that incorporates sets of progressively larger size [12-16]. Several different approaches have been taken, one of which included a simple exponential [12], which was expanded to a more complicated mixed Gaussian/exponential expression [17]. Also tested has been an expansion in inverse powers of the quantum number $\ell[16,18-20]$, and an inverse power expression involving three parameters [21]. A fuller assortment of various means to extrapolate to the complete basis set limit has been reviewed [22,23], along with an assessment of the reliability of each, with particular reference to atomization energies. 
The study of intermolecular interactions represents a promising area for the application of such extrapolation schemes. The strength of noncovalent forces can be difficult to compute accurately, as it represents a much weaker interaction than intramolecular forces, so the ability to apply very high-accuracy methods would be most welcome. There are impediments, however, to the ready application of extrapolation schemes. In the first place, the systems of interest are typically of moderate to large size, which prohibits the application of high levels of correlation or the very large basis sets required as input in some of these schemes. There is also the issue of basis set superposition, an artifact which can contaminate interaction energies to a large degree. However, these problems are not insurmountable, as for example Feller's consideration of the water dimer [12] where he was able to arrive at an accurate complete basis set, full CI H-bond strength, which was appropriately corrected for basis set superposition error. He has shown also that extrapolation procedures can be applied to a larger system such as the benzene-water complex [24]. More recent studies have examined the basis set convergence of electron correlation via the random phase approach [25] and concluded that inclusion of counterpoise correction without extrapolation is insufficient, again for the water dimer and water-benzene interactions.

While H-bonds of the latter sort have been the subject of a number of high-accuracy extrapolation schemes, there have been few attempts to apply this strategy to related noncovalent interactions. In particular, the pnicogen bond between $\mathrm{P}$ and/or $\mathrm{N}$ atoms has been shown of late to represent a very significant source of intermolecular attraction [26-30]. Work from this group [31-42] has pointed to two major factors that contribute to the stability of the pnicogen bond. There is first a transfer of charge from the lone pair of one pnicogen atom to a $\sigma^{*}$ antibonding orbital of the other, much like is found in H-bonds. In contrast to a H-bond, however, the B 
atom (bound to the $\mathrm{P}$ ) is turned away from the donor $\mathrm{N}$ lone pair, so that it is the lobe of the $\mathrm{P}-\mathrm{B}$ $\sigma^{*}$ orbital that is located near the $\mathrm{P}$ atom that is the recipient of this charge. This induction-type energy is supplemented by an attractive electrostatic component that arises from the overlap of the negatively charged region around the $\mathrm{N}$ lone pair and a positively charged area on the partner molecule.

Given the newcomer status of the pnicogen bond to the pantheon of noncovalent forces, it is desirable to have accurate measures of its stability, so that it may be reliably compared to other, related interactions, such as hydrogen and halogen bonds. The relevant systems are too large to perform full, or nearly full CI calculations, and there are curbs on the size of the basis sets that might be applied. It is for this reason that the concept of extrapolation to complete basis set results becomes a very attractive option. A method that was proposed by Truhlar [43] seems particularly worthy of examination. This approach rests on the idea of computation with both double and triple-valence basis sets, both of which are feasible for the systems of interest. Moreover, it has been developed so as to be compatible with MP2, CCSD, and CCSD(T) correlation methods, also viable for these systems.

The present work represents a test of this extrapolation scheme for the pnicogen bond between $\mathrm{P}$ and $\mathrm{N}$ atoms. So as to cover a range of interaction energies, a set of systems is considered here that was shown earlier [34] to extend from 1 to $7 \mathrm{kcal} / \mathrm{mol}$. In particular, the systems investigated are $\mathrm{BH}_{2} \mathrm{P} \cdots \mathrm{NH}_{3}$, where the substituent $\mathrm{B}=\mathrm{H}, \mathrm{CH}_{3}, \mathrm{NH}_{2}, \mathrm{CF}_{3}, \mathrm{OH}, \mathrm{Cl}, \mathrm{F}$, and $\mathrm{NO}_{2}$. The data permit an evaluation of the accuracy of the double and triple-valence basis set results, without extrapolation. Also tested is the error incurred by failure to correct for basis set superposition error at each level. There is also the opportunity to examine the accuracy of the 
MP2 method, with and without extrapolation to a complete basis set. The ideas are finally tested on a prototypical $\mathrm{H}$-bonded system, in this case the water dimer.

\section{METHODS}

All calculations were carried out with the Gaussian-09 package of codes [44]. The geometries of all species, complexes and monomers, were optimized at the MP2/aug-cc-pVDZ level. Energies were computed at the SCF, MP2, and CCSD(T) levels with both the latter basis set and also with aug-cc-pVTZ. Following Truhlar [43], the complete or infinite basis set limit for the Hartree-Fock energy was taken as

$$
\underset{\mathrm{CBS}}{\mathrm{HF}}=\frac{3^{\alpha}}{3^{\alpha}-2^{\alpha}} \mathrm{E}_{3}^{\mathrm{HF}}-\frac{2^{\alpha}}{3^{\alpha}-2^{\alpha}} \mathrm{E}_{2}^{\mathrm{HF}}
$$

where $E_{2}$ and $E_{3}$ refer to the energies computed with the aug-cc-pVDZ and aug-cc-pVTZ basis sets, respectively. A similar expression, with exponent $\alpha$ replaced by $\beta$, was used for the correlation energy:

$$
\mathrm{E}_{\mathrm{CBS}}^{\mathrm{corr}}=\frac{3^{\beta}}{3^{\beta}-2^{\beta}} \mathrm{E}_{3}^{\mathrm{corr}}-\frac{2^{\beta}}{3^{\beta}-2^{\beta}} \mathrm{E}_{2}^{\text {corr }}
$$

Truhlar had optimized the SCF $\alpha$ exponent to be 3.4, whereas $\beta$ was equal to 2.2 for MP2 and 2.4 for $\operatorname{CCSD}(\mathrm{T})$, so these values were applied here. CBS energies were computed for all species, monomers and complexes, via the prescriptions above. The extrapolated MP2 and $\operatorname{CCSD}(\mathrm{T})$ energies of each species were calculated by adding the appropriate $\mathrm{CBS}^{\mathrm{corr}}$ to the extrapolated HF energy. $\Delta \mathrm{E}$ was taken as the difference between the energy of the optimized dimer on one hand, and the sum of the energies of the individual monomers on the other. Counterpoise corrections for the basis set superposition error were formulated via the BoysBernardi procedure [45]. These corrections were added to the energies of the complexes at both 
the $\mathrm{D}$ and $\mathrm{T}$ levels, and were thereby included in the extrapolation of the complex energies to the complete basis set limit.

\section{RESULTS}

The binding energies computed for each complex are reported in Table 1 at the SCF, MP2, and $\operatorname{CCSD}(\mathrm{T})$ levels. At each level, the binding energy is presented for the aug-cc-pVDZ (D) and aug-cc-pVTZ (T) basis sets, followed by the extrapolated CBS result. Similar data are displayed in Table 2, with the additional feature that counterpoise corrections are added to each complexation energy. It is for this reason that the binding energies in Table 2 are somewhat smaller than the uncorrected values in Table 1.

Due to the importance of correlation to the binding of many of these complexes, the SCF binding energies are significantly smaller than the MP2 and $\operatorname{CCSD}(\mathrm{T})$ values. In fact, slightly negative binding energies are obtained for the most weakly bound $\mathrm{CH}_{3} \mathrm{H}_{2} \mathrm{P} \cdots \mathrm{NH}_{3}$ complex, indicating an unbound structure without correlation effects. In most cases, the interaction energy increases in the order $\mathrm{B}=\mathrm{CH}_{3}<\mathrm{H}<\mathrm{NH}_{2}<\mathrm{CF}_{3}<\mathrm{OH}<\mathrm{Cl}<\mathrm{F}<\mathrm{NO}_{2}$ at any level of theory, and with either basis set, although small deviations do occur. One such deviation may be noted for $\mathrm{B}=\mathrm{CF}_{3}$ and $\mathrm{OH}$. At the $\mathrm{SCF}$ level, the former makes for stronger binding, a trend that is reversed when correlation is included.

Another interesting pattern is related to the inclusion of counterpoise corrections. When these corrections are not included, as in Table 1, the $\mathrm{T}$ binding energies are uniformly smaller than the D results. This pattern is true at the SCF, MP2, and $\operatorname{CCSD}(\mathrm{T})$ levels. It is therefore not surprising to note that the extrapolated values are also smaller than D data. An opposite trend is observed in Table 2. With the inclusion of counterpoise corrections, the $\mathrm{T}$ binding energies are larger than the D values, and the CBS quantities even more so. (The latter pattern is true only of 
the correlated MP2 and CCSD(T) values, not SCF.) In other words, correlated MP2/aug-ccpVDZ binding energies represent an overestimate if one ignores basis set superposition error, but a small underestimate occurs when this error is corrected.

\section{Accuracy of Various Methods}

As fully extrapolated calculations may not always be possible, nor might $\operatorname{CCSD}(\mathrm{T})$ be a viable option for all systems, it would be useful to have some estimate as to the level of accuracy that might be expected for each theoretical approach. For purposes of comparison, the extrapolated $\operatorname{CCSD}(\mathrm{T})$ data, with counterpoise corrections, from the last column of Table 2 are taken as the benchmark. Tables 3 and 4 report the errors intrinsic to each level of theory in several different manners. The mean error (ME) permits a user to estimate a possible correction that could be made to computed data that might allow an approximation to the correct value. For example, the negative values of ME for all of the SCF data are indicative of the fact that the HF approximation consistently underestimates the binding energy. The mean absolute error (MAE) is also an average of errors, but does not differentiate between over and under estimates, targeting instead the absolute error of each approach. It is for this reason that the extrapolated ME and MAE results in the last column of Table 3, uncorrected for basis set superposition, are so different. Since some of the errors are positive, and others negative, their cancellation leads to a very small mean error of only $0.03 \mathrm{kcal} / \mathrm{mol}$. However, this small quantity might lead to a misleading level of comfort, since the absolute values of the errors average to the much larger amount of $0.20 \mathrm{kcal} / \mathrm{mol}$. The last row of Tables 3 and 4 refer to the root mean square deviation, a perhaps more common measure of accuracy. These quantities tend to be similar to, but slightly larger than, the mean absolute errors in the preceding row. 
Examination of the data in Tables 3 and 4 make it immediately obvious that SCF calculations provide severe underestimates of the binding energies, with either basis set or with extrapolation, and with or without counterpoise correction. MP2 energies are closer to the benchmark, within about $1 \mathrm{kcal} / \mathrm{mol}$ with the aug-cc-pDVZ basis set. The error is considerably smaller for the triple-valence set, and is not significantly improved further by basis set extrapolation. It is interesting to note that the MP2/aug-cc-pDVZ binding energies are uniformly too high (by nearly $1 \mathrm{kcal} / \mathrm{mol}$ ) without counterpoise correction, but underestimate the benchmark by a like amount if corrected. When MP2 data are counterpoise corrected and extrapolated, the binding energies are accurate within about $0.25 \mathrm{kcal} / \mathrm{mol}$. Turning finally to $\operatorname{CCSD}(\mathrm{T})$ data, even when uncorrected for BSSE, the CBS data are quite close to the benchmark, within about $0.2 \mathrm{kcal} / \mathrm{mol}$. It is interesting to observe that the non-extrapolated $\operatorname{CCSD}(\mathrm{T})$ data are only of moderate accuracy, even with the triple-valence set that contains an error of roughly $0.5 \mathrm{kcal} / \mathrm{mol}$.

Based on the data in Tables 3 and 4, one can conclude that SCF data are unacceptable, with any basis set. MP2, however, offers a very serviceable approximation, particularly with the augcc-pVTZ basis set, and with counterpoise corrections included. Performing CCSD(T) calculations will improve on this prescription only if the basis set is fully extrapolated, again with counterpoise corrections. Indeed, without such extrapolation, $\operatorname{CCSD}(\mathrm{T})$ binding energies are inferior to those obtained at the more economical MP2 level.

Fig 1 provides an instructive graphical view of the asymptotic behavior of each level of theory. The system taken for illustrative purposes is the $\mathrm{FH}_{2} \mathrm{P} \cdots \mathrm{NH}_{3}$ complex. The broken curves represent the binding energies prior to counterpoise correction, and the appropriately corrected values are illustrated by the solid curves. There are several patterns that are evident from the figure. First of all, the uncorrected values are expectedly too high compared to the solid 
curves. Note that the uncorrected binding energies become smaller as the basis set is enlarged, while the corrected values behave in the opposite fashion, growing with larger basis. The differences between the uncorrected and corrected quantities diminish as the basis set is enlarged. As there is still a clear distinction at the CBS limit, counterpoise correction remains necessary for quantitative accuracy. It may also be observed that the MP2 binding energies are uniformly higher than $\operatorname{CCSD}(\mathrm{T})$ quantities, with or without counterpoise correction. As an interesting aside, in the case of $\mathrm{FH}_{2} \mathrm{P} \cdots \mathrm{NH}_{3}$, the corrected MP2/aug-cc-pVDZ binding energy is quite similar to the extrapolated $\operatorname{CCSD}(\mathrm{T})$ value.

\section{Potential Application to H-bonds}

Given the conclusions above, it is natural to wonder how these various levels of theory stack up for molecular interactions other than the pnicogen bonds considered here. As one prototypical example, similar calculations were carried out for the water dimer. The results are reported in the last row of Tables 1 and 2. The CBS extrapolated CCSD(T) binding energy, with counterpoise corrections, may be seen to be $4.97 \mathrm{kcal} / \mathrm{mol}$, taken as the benchmark. As in the pnicogen-bonded systems, the failure to include correlation results in a sizable underestimate of this quantity, by roughly $1.5 \mathrm{kcal} / \mathrm{mol}$. Considering the MP2 and $\operatorname{CCSD}(\mathrm{T})$ correlated data in Table 1, it is clear that failure to include counterpoise corrections leads to an overestimate with any basis set. As in the case of the pnicogen bonds, the MP2 approach provides a very good approximation of the benchmark value, particularly when applied to the triple-valence set; this $0.26 \mathrm{kcal} / \mathrm{mol}$ error nearly vanishes altogether after basis set extrapolation.

In summary, the straightforward scheme which extrapolates double and triple-valence sets to the complete basis set limit seems quite well suited to the noncovalent interactions of interest here. This scheme offers a viable procedure, which extrapolates smoothly to an infinite-size 
basis set. Within the context of the $\mathrm{BH}_{2} \mathrm{P} \cdots \mathrm{NH}_{3}$ complexes considered here, all levels of theory provide a similar ordering of the binding energy with respect to the substituent B. Not unexpectedly, SCF values are much too small, but MP2 data are similar to $\operatorname{CCSD}(\mathrm{T})$ quantities. Even when extrapolated, it remains necessary to include counterpoise corrections to the basis set superposition error. On a statistical basis, if the full extrapolation to the $\operatorname{CCSD}(\mathrm{T})$ result is not feasible, the best option appears to be extrapolation of (counterpoise-corrected) MP2 data, the mean absolute error of which is $0.25 \mathrm{kcal} / \mathrm{mol}$. Almost as accurate is the unextrapolated MP2/aug-cc-pVTZ level, with an error of $0.27 \mathrm{kcal} / \mathrm{mol}$. Testing of this procedure on the $\mathrm{H}$ bonded water dimer provides an optimistic assessment that this scheme ought to be useful on these noncovalent interactions as well. 


\section{REFERENCES}

[1] M.R. Nyden, G.A. Petersson, Complete basis set correlation energies. I. The asymptotic convergence of pair natural orbital expansions, J. Chem. Phys. 75 (1981) 1843-1862.

[2] F.B. Brown, D.G. Truhlar, A new semi-empirical method of correcting large-scale configuration interaction calculations for incomplete dynamic correlation of electrons, Chem. Phys. Lett. 117 (1985) 307-313.

[3] G.A. Petersson, A.K. Lee, A. Bennett, Complete basis set correlation energies. III. The total correlation energy of the neon atom, J. Chem. Phys. 83 (1985) 5105-5128.

[4] M.S. Gordon, D.G. Truhlar, Scaling all correlation energy in perturbation theory calculations of bond energies and barrier heights, J. Am. Chem. Soc. 108 (1986) 54125419.

[5] M.S. Gordon, K.A. Nguyen, D.G. Truhlar, Parameters for scaling the correlation energy of the bonds silicon-hydrogen, phosphorus-hydrogen- sulfur-hydrogen, and chlorinehydrogen and application to the reaction of silyl radical with silane, J. Phys. Chem. 93 (1989) 7356-7358.

[6] G.A. Petersson, M.A. Al-Laham, A complete basis set model chemistry. II. Open-shell systems and the total energies of the first-row atoms, J. Chem. Phys. 94 (1991) 60816090.

[7] P.E.M. Siegbahn, M.R.A. Blomberg, M. Svensson, PCI-X, a parametrized correlation method containing a single adjustable parameter X, Chem. Phys. Lett. 223 (1994) 35-45.

[8] J.A. Montgomery, J.W. Ochterski, G.A. Petersson, A complete basis set model chemistry. IV. An improved atomic pair natural orbital method, J. Chem. Phys. 101 (1994) 5900-5909.

[9] I. Rossi, D.G. Truhlar, Improved general scaling factors and systematic tests of the SAC method for estimating correlation energies of molecules, Chem. Phys. Lett. 234 (1995) 64-70.

[10] S.S. Xantheas, On the importance of the fragment relaxation energy terms in the estimation of the basis set superposition error correction to the intermolecular interaction energy, J. Chem. Phys. 104 (1996) 8821-8824.

[11] J.W. Ochterski, G.A. Petersson, J.A. Montgomery, A complete basis set model chemistry. V. Extensions to six or more heavy atoms, J. Chem. Phys. 104 (1996) 25982619.

[12] D. Feller, Application of systematic sequences of wave functions to the water dimer, J. Chem. Phys. 96 (1992) 6104-6114.

[13] K.A. Peterson, T.H. Dunning, Intrinsic Errors in Several ab Initio Methods: The Dissociation Energy of $\mathrm{N}_{2}$, J. Phys. Chem. 99 (1995) 3898-3901.

[14] J.M.L. Martin, Ab initio total atomization energies of small molecules - towards the basis set limit, Chem. Phys. Lett. 259 (1996) 669-678.

[15] J.M.L. Martin, P.R. Taylor, Benchmark quality total atomization energies of small polyatomic molecules, J. Chem. Phys. 106 (1997) 8620-8623.

[16] A. Halkier, T. Helgaker, P. Jørgensen, W. Klopper, H. Koch, J. Olsen, A.K. Wilson, Basis-set convergence in correlated calculations on $\mathrm{Ne}, \mathrm{N}_{2}$, and $\mathrm{H}_{2} \mathrm{O}$, Chem. Phys. Lett. 286 (1998) 243-252.

[17] K.A. Peterson, D.E. Woon, J. Thom H. Dunning, Benchmark calculations with correlated molecular wave functions. IV. The classical barrier height of the $\mathrm{H}+\mathrm{H}_{2}$ to $\mathrm{H}_{2}+\mathrm{H}$ reaction, J. Chem. Phys. 100 (1994) 7410-7415. 
[18] D.P. Carroll, H.J. Silverstone, R.M. Metzger, Piecewise polynomial configuration interaction natural orbital study of $1 \mathrm{~s}^{2}$ helium, J. Chem. Phys. 71 (1979) 4142-4163.

[19] R.N. Hill, Rates of convergence and error estimation formulas for the Rayleigh-Ritz variational method, J. Chem. Phys. 83 (1985) 1173-1196.

[20] W. Kutzelnigg, J.D. Morgan, Rates of convergence of the partial-wave expansions of atomic correlation energies, J. Chem. Phys. 96 (1992) 4484-4508.

[21] J.M.L. Martin, T.J. Lee, Accurate ab initio quartic force field and vibrational frequencies of the $\mathrm{NH}_{4}{ }^{+}$ion and its deuterated forms, Chem. Phys. Lett. 258 (1996) 129-135.

[22] D. Feller, K.A. Peterson, Re-examination of atomization energies for the Gaussian-2 set of molecules, J. Chem. Phys. 110 (1999) 8384-8396.

[23] D. Feller, K.A. Peterson, J.G. Hill, On the effectiveness of CCSD(T) complete basis set extrapolations for atomization energies, J. Chem. Phys. 135 (2011) 044102.

[24] D. Feller, Strength of the benzene-water hydrogen bond, J. Phys. Chem. A. 103 (1999) 7558-7561.

[25] H. Eshuis, F. Furche, Basis set convergence of molecular correlation energy differences within the random phase approximation, J. Chem. Phys. 134 (2012) 084105.

[26] S. Zahn, R. Frank, E. Hey-Hawkins, B. Kirchner, Pnicogen bonds: A new molecular linker?, Chem. Eur. J. 17 (2011) 6034-6038.

[27] M. Bühl, P. Kilian, J.D. Woollins, Prediction of a new delocalised bonding motif between group 15 or group 16 atoms, ChemPhysChem. 12 (2011) 2405-2408.

[28] J.E.D. Bene, I. Alkorta, G. Sanchez-Sanz, J. Elguero, ${ }^{31} \mathrm{P}-{ }^{31} \mathrm{P}$ spin-spin coupling constants for pnicogen homodimers, Chem. Phys. Lett. 512 (2011) 184-187.

[29] J.E.D. Bene, I. Alkorta, G. Sanchez-Sanz, J. Elguero, Structures, energies, bonding, and NMR properties of pnicogen complexes $\mathrm{H}_{2} \mathrm{XP}: \mathrm{NXH}_{2}\left(\mathrm{X}=\mathrm{H}, \mathrm{CH}_{3}, \mathrm{NH}_{2}, \mathrm{OH}, \mathrm{F}, \mathrm{Cl}\right), \mathrm{J}$. Phys. Chem. A. 115 (2011) 13724-13731.

[30] J.E.D. Bene, I. Alkorta, G. Sanchez-Sanz, J. Elguero, Structures, binding energies, and spin-spin coupling constants of geometric isomers of pnicogen homodimers $(\mathrm{PHFX})_{2}, \mathrm{X}$ $=\mathrm{F}, \mathrm{Cl}, \mathrm{CN}, \mathrm{CH}_{3}$, NC, J. Phys. Chem. A. 116 (2012) 3056-3060.

[31] S. Scheiner, Weak H-bonds. Comparisons of $\mathrm{CH}^{\cdots} \mathrm{O}^{\mathrm{O}}$ to $\mathrm{NH}^{\cdots} \mathrm{O}$ in proteins and $\mathrm{PH}{ }^{\cdots} \mathrm{N}$ to direct P"'N interactions, Phys. Chem. Chem. Phys. 13 (2011) 13860-13872.

[32] S. Scheiner, A new noncovalent force: Comparison of P'*N Interaction with hydrogen and halogen bonds, J. Chem. Phys. 134 (2011) 094315.

[33] S. Scheiner, On the properties of $X^{\cdots} \mathrm{N}$ noncovalent interactions for first-, second- and third-row X atoms, J. Chem. Phys. 134 (2011) 164313.

[34] S. Scheiner, Effects of substituents upon the P'*N noncovalent interaction: The limits of its strength, J. Phys. Chem. A. 115 (2011) 11202-11209.

[35] S. Scheiner, Effects of multiple substitution upon the $\mathrm{P}{ }^{*} \mathrm{~N}$ noncovalent interaction, Chem. Phys. 387 (2011) 79-84.

[36] S. Scheiner, Can two trivalent $\mathrm{N}$ atoms engage in a direct $\mathrm{N} \cdots \mathrm{N}$ noncovalent interaction?, Chem. Phys. Lett. 514 (2011) 32-35.

[37] U. Adhikari, S. Scheiner, The S'*N noncovalent interaction: Comparison with hydrogen and halogen bonds, Chem. Phys. Lett. 514 (2011) 36-39.

[38] U. Adhikari, S. Scheiner, Comparison of P'” $D(D=P, N)$ with other noncovalent bonds in molecular aggregates, J. Chem. Phys. 135 (2011) 184306.

[39] S. Scheiner, U. Adhikari, Abilities of different electron donors (D) to engage in a P"*D noncovalent interaction, J. Phys. Chem. A. 115 (2011) 11101-11110. 


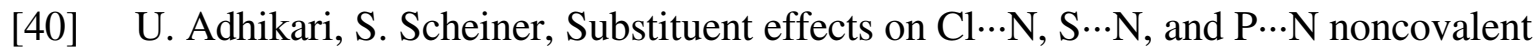
bonds, J. Phys. Chem. A. (2012) in press.

[41] U. Adhikari, S. Scheiner, Sensitivity of pnicogen, chalcogen, halogen and H-bonds to angular distortions, Chem. Phys. Lett. (2012) in press.

[42] U. Adhikari, S. Scheiner, Effects of carbon chain substituent on the P $\cdots \mathrm{N}$ noncovalent bond, Chem. Phys. Lett. (2012) in press.

[43] D.G. Truhlar, Basis-set extrapolation, Chem. Phys. Lett. 294 (1998) 45-48.

[44] M.J. Frisch, G.W. Trucks, H.B. Schlegel, G. E. Scuseria, M.A. Robb, J.R. Cheeseman, G. Scalmani, V. Barone, B. Mennucci, G.A. Petersson, H. Nakatsuji, M. Caricato, X. Li, H.P. Hratchian, A.F. Izmaylov, J. Bloino, G. Zheng, J.L. Sonnenberg, M. Hada, M. Ehara, K. Toyota, R. Fukuda, J. Hasegawa, M. Ishida, T. Nakajima, Y. Honda, O. Kitao, H. Nakai, T. Vreven, J.A. Montgomery, J.E. Peralta, F. Ogliaro, M. Bearpark, J.J. Heyd, E. Brothers, K.N. Kudin, V.N. Staroverov, T. Keith, R. Kobayashi, J. Normand, K. Raghavachari, A. Rendell, J.C. Burant, S.S. Iyengar, J. Tomasi, M. Cossi, N. Rega, J.M. Millam, M. Klene, J.E. Knox, J.B. Cross, V. Bakken, C. Adamo, J. Jaramillo, R. Gomperts, R.E. Stratmann, O. Yazyev, A.J. Austin, R. Cammi, C. Pomelli, J.W. Ochterski, R.L. Martin, K. Morokuma, V.G. Zakrzewski, G.A. Voth, P. Salvador, J.J. Dannenberg, S. Dapprich, A.D. Daniels, O. Farkas, J.B. Foresman, J.V. Ortiz, J. Cioslowski, D.J. Fox, Gaussian 09. Gaussian, Inc, Wallingford CT, 2009.

[45] S.F. Boys, F. Bernardi, The calculation of small molecular interactions by the differences of separate total energies. Some procedures with reduced errors, Mol. Phys. 19 (1970) 553-566. 
Table 1. Binding energies $(\mathrm{kcal} / \mathrm{mol})$ calculated for $\mathrm{BH}_{2} \mathrm{P} \cdots \mathrm{NH}_{3}$ and water dimer with the augcc-pVnZ basis set $(\mathrm{n}=\mathrm{D}, \mathrm{T})$.

\begin{tabular}{|l|l|l|l|l|c|c|c|c|c|}
\hline B & \multicolumn{3}{|c|}{ SCF } & \multicolumn{3}{c|}{ MP2 } & \multicolumn{3}{c|}{ CCSD(T) } \\
\hline & \multicolumn{1}{|c|}{ D } & \multicolumn{1}{|c|}{ T } & CBS & D & T & CBS & D & T & CBS \\
\hline $\mathrm{CH}_{3}$ & -0.31 & -0.03 & 0.07 & 2.16 & 1.90 & 1.83 & 2.11 & 1.84 & 1.75 \\
\hline $\mathrm{H}$ & 0.41 & 0.22 & 0.16 & 2.05 & 1.95 & 1.95 & 1.96 & 1.86 & 1.86 \\
\hline $\mathrm{NH}_{2}$ & 0.71 & 0.16 & -0.03 & 3.19 & 2.73 & 2.61 & 3.09 & 2.61 & 2.47 \\
\hline $\mathrm{CF}_{3}$ & 2.36 & 1.75 & 1.54 & 4.58 & 4.08 & 3.95 & 4.53 & 4.00 & 3.84 \\
\hline $\mathrm{OH}$ & 1.55 & 0.66 & 0.36 & 5.31 & 4.64 & 4.50 & 5.01 & 4.32 & 4.14 \\
\hline $\mathrm{Cl}$ & 2.92 & 1.91 & 1.56 & 6.99 & 6.25 & 6.10 & 6.50 & 5.78 & 5.62 \\
\hline $\mathrm{F}$ & 3.14 & 2.15 & 1.82 & 7.89 & 7.32 & 7.27 & 7.39 & 6.81 & 6.72 \\
\hline $\mathrm{NO}_{2}$ & 5.43 & 4.76 & 4.54 & 8.30 & 8.04 & 8.09 & 8.05 & 7.77 & 7.79 \\
\hline$\left(\mathrm{H}_{2} \mathrm{O}\right)_{2}$ & 3.68 & 3.49 & 3.42 & 5.26 & 5.18 & 5.19 & 5.30 & 5.21 & 5.21 \\
\hline
\end{tabular}

Table 2. Binding energies $(\mathrm{kcal} / \mathrm{mol})$ calculated for $\mathrm{BH}_{2} \mathrm{P} \cdots \mathrm{NH}_{3}$ and water dimer with the augcc-pVnZ basis set $(\mathrm{n}=\mathrm{D}, \mathrm{T})$, all results corrected by counterpoise procedure.

\begin{tabular}{|l|l|l|l|l|c|c|c|c|c|}
\hline B & \multicolumn{3}{|c|}{ SCF } & \multicolumn{3}{c|}{ MP2 } & \multicolumn{3}{c|}{ CCSD(T) } \\
\hline & \multicolumn{1}{|c|}{ D } & T & CBS & D & T & CBS & D & T & CBS \\
\hline $\mathrm{CH}_{3}$ & -0.05 & -0.03 & -0.02 & 1.33 & 1.53 & 1.66 & 1.25 & 1.48 & 1.61 \\
\hline $\mathrm{H}$ & 0.17 & 0.17 & 0.17 & 1.18 & 1.61 & 1.90 & 1.08 & 1.53 & 1.81 \\
\hline $\mathrm{NH}_{2}$ & 0.17 & 0.09 & 0.07 & 2.09 & 2.31 & 2.49 & 1.96 & 2.22 & 2.39 \\
\hline $\mathrm{CF}$ & 1.79 & 1.66 & 1.62 & 2.82 & 3.58 & 4.14 & 2.73 & 3.51 & 4.03 \\
\hline $\mathrm{OH}$ & 0.94 & 0.55 & 0.42 & 3.98 & 4.17 & 4.44 & 3.63 & 3.87 & 4.11 \\
\hline $\mathrm{Cl}$ & 2.22 & 1.80 & 1.66 & 4.63 & 5.51 & 6.27 & 4.08 & 5.07 & 5.78 \\
\hline $\mathrm{F}$ & 2.42 & 1.91 & 1.74 & 6.18 & 6.35 & 6.64 & 5.61 & 5.86 & 6.14 \\
\hline $\mathrm{NO}_{2}$ & 4.66 & 4.66 & 4.66 & 5.81 & 7.35 & 8.42 & 5.49 & 7.12 & 8.12 \\
\hline$\left(\mathrm{H}_{2} \mathrm{O}\right)_{2}$ & 3.44 & 3.41 & 3.40 & 4.43 & 4.71 & 4.92 & 4.39 & 4.74 & 4.97 \\
\hline
\end{tabular}


Table 3. Errors (kcal/mol) incurred by application of each method, uncorrected for basis set superposition error, to interaction energy of $\mathrm{BH}_{2} \mathrm{P} \cdots \mathrm{NH}_{3}$.

\begin{tabular}{|l|c|c|c|c|c|c|c|c|c|}
\hline & \multicolumn{3}{|c|}{ SCF } & \multicolumn{3}{c|}{ MP2 } & \multicolumn{3}{c|}{ CCSD(T) } \\
\hline & D & T & CBS & D & T & CBS & D & T & CBS \\
\hline ME $^{\mathrm{a}}$ & -2.22 & -2.80 & -3.00 & 0.81 & 0.37 & 0.29 & 0.58 & 0.13 & 0.03 \\
\hline MAE $^{\mathrm{b}}$ & 2.22 & 2.80 & 3.00 & 0.81 & 0.39 & 0.32 & 0.60 & 0.22 & 0.20 \\
\hline RMS $^{\mathrm{c}}$ & 2.30 & 2.95 & 3.17 & 0.96 & 0.52 & 0.45 & 0.70 & 0.30 & 0.26 \\
\hline
\end{tabular}

${ }^{\mathrm{a}}$ mean error

${ }^{b}$ mean absolute error

${ }^{c}$ root mean square error

Table 4. Errors (kcal/mol) incurred by application of each method, corrected for basis set superposition error, to interaction energy of $\mathrm{BH}_{2} \mathrm{P} \cdots \mathrm{NH}_{3}$.

\begin{tabular}{|l|c|c|c|c|c|c|c|c|c|}
\hline & \multicolumn{3}{|c|}{ SCF } & \multicolumn{3}{c|}{ MP2 } & \multicolumn{3}{c|}{ CCSD(T) } \\
\hline & $\mathrm{D}$ & $\mathrm{T}$ & $\mathrm{CBS}$ & $\mathrm{D}$ & $\mathrm{T}$ & $\mathrm{CBS}$ & $\mathrm{D}$ & $\mathrm{T}$ & CBS \\
\hline ME $^{\mathrm{a}}$ & -2.71 & -2.90 & -2.96 & -0.75 & -0.20 & 0.25 & -1.02 & -0.42 & 0 \\
\hline MAE $^{\mathrm{b}}$ & 2.71 & 2.90 & 2.96 & 0.76 & 0.27 & 0.25 & 1.02 & 0.42 & 0 \\
\hline RMS $^{\mathrm{c}}$ & 2.83 & 3.06 & 3.13 & 1.04 & 0.35 & 0.30 & 1.27 & 0.50 & 0 \\
\hline
\end{tabular}




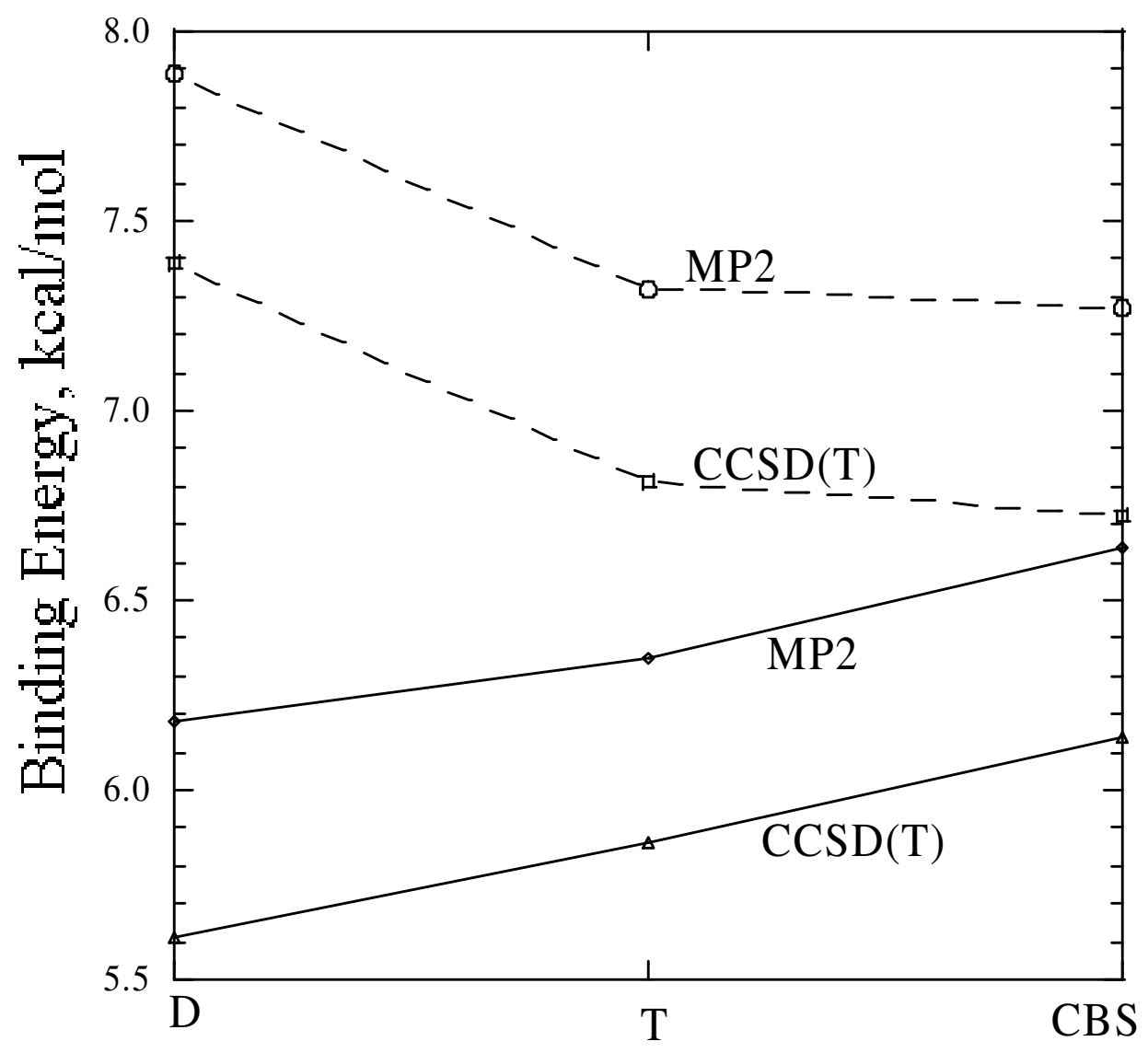

Fig. 1. Binding energy of the $\mathrm{FH}_{2} \mathrm{P} \cdots \mathrm{NH}_{3}$ complex computed at various levels. Broken curves have not been corrected for basis set superposition error, solid curves incorporate the counterpoise correction. The $\mathrm{D}$ and $\mathrm{T}$ designations refer to aug-cc-pVDZ and aug-ccpVTZ basis sets, respectively. 